PROCEEDINGS OF THE

AMERICAN MATHEMATICAL SOCIETY

Volume 133, Number 6, Pages 1657-1664

S 0002-9939(05)07841-X

Article electronically published on January 13, 2005

\title{
A GENERAL FUNCTIONAL EQUATION AND ITS STABILITY
}

\author{
JOHN A. BAKER
}

(Communicated by Jonathan M. Borwein)

\begin{abstract}
Suppose that $V$ and $B$ are vector spaces over $\mathbb{Q}, \mathbb{R}$ or $\mathbb{C}$ and $\alpha_{0}, \beta_{0}, \ldots, \alpha_{m}, \beta_{m}$ are scalar such that $\alpha_{j} \beta_{k}-\alpha_{k} \beta_{j} \neq 0$ whenever $0 \leq j<$ $k \leq m$. We prove that if $f_{k}: V \rightarrow B$ for $0 \leq k \leq m$ and

$$
\sum_{k=0}^{m} f_{k}\left(\alpha_{k} x+\beta_{k} y\right)=0 \quad \text { for all } x, y \in V,
$$

then each $f_{k}$ is a "generalized" polynomial map of "degree" at most $m-1$.

In case $V=\mathbb{R}^{n}$ and $B=\mathbb{C}$ we show that if some $f_{k}$ is bounded on a set of positive inner Lebesgue measure, then it is a genuine polynomial function.

Our main aim is to establish the stability of $(*)$ (in the sense of Ulam) in case $B$ is a Banach space.

We also solve a distributional analogue of $(*)$ and prove a mean value theorem concerning harmonic functions in two real variables.
\end{abstract}

\section{BLANKET ASSUMPTIONS}

Throughout this paper $F=\mathbb{Q}, \mathbb{R}$ or $\mathbb{C}, V$ and $B$ are vector spaces over $F, m \in \mathbb{N}$, and $\alpha_{0}, \beta_{0}, \ldots, \alpha_{m}, \beta_{m}$ are given members of $F$. Our aim is to study the functional equation

$$
\sum_{k=0}^{m} f_{k}\left(\alpha_{k} x+\beta_{k} y\right)=0 \text { for all } x, y \in V,
$$

where the functions $f_{k}: V \rightarrow B$ may be thought of as the unknowns. The stability of $(*)$ (in the sense of Ulam) is of principal interest.

\section{BACKGROUND RESULTS}

A function $a: V \rightarrow B$ is said to be additive provided $a(x+y)=a(x)+a(y)$ for all $x, y \in V$; in this case it is easily seen that $a(r x)=r a(x)$ for all $x \in V$ and all $r \in \mathbb{Q}$.

If $k \in \mathbb{N}$ and $a: V^{k} \rightarrow B$, then we say that $a$ is $k$-additive provided it is additive in each variable; we say that $a$ is symmetric provided $a\left(x_{1}, x_{2}, \ldots, x_{k}\right)=$ $a\left(y_{1}, y_{2}, \ldots, y_{k}\right)$ whenever $x_{1}, x_{2}, \ldots, x_{k} \in V$ and $\left(y_{1}, y_{2}, \ldots, y_{k}\right)$ is a permutation of $\left(x_{1}, x_{2}, \ldots, x_{k}\right)$.

Received by the editors April 25, 2003

2000 Mathematics Subject Classification. Primary 39B72, 39B52; Secondary 39B05.

Key words and phrases. Functional equation, stability.

(C)2005 American Mathematical Society Reverts to public domain 28 years from publication 
If $k \in \mathbb{N}$ and $a: V^{k} \rightarrow B$ is symmetric and $k$-additive, let $a^{*}(x)=a(x, x, \ldots, x)$ for $x \in V$ and note that $a^{*}(r x)=r^{k} a(x)$ whenever $x \in V$ and $r \in \mathbb{Q}$. Such a function $a^{*}$ will be called a monomial function of degree $k$ (assuming $a^{*} \not \equiv 0$ ).

A function $p: V \rightarrow B$ is called a generalized polynomial (GP) function of degree $m \in \mathbb{N}$ provided there exist $a_{0} \in B$ and symmetric $k$-additive functions $a_{k}: V^{k} \rightarrow B$ (for $1 \leq k \leq m$ ) such that

$$
p(x)=a_{0}+\sum_{k=1}^{m} a_{k}^{*}(x) \quad \text { for all } x \in V,
$$

and $a_{m}^{*} \not \equiv 0$. In this case

$$
p(r x)=a_{0}+\sum_{k=1}^{m} r^{k} a_{k}^{*}(x) \quad \text { for } x \in V \text { and } r \in \mathbb{Q} .
$$

Let $B^{V}$ denote the vector space (over $F$ ) consisting of all maps from $V$ into $B$. For $h \in V$ define the linear "difference" operator $\Delta_{h}$ on $B^{V}$ by

$$
\underset{h}{\Delta} f(x)=f(x+h)-f(x) \text { for } f \in B^{V} \text { and } x \in V .
$$

Notice that these difference operators commute $\left(\underset{h_{1} h_{2}}{\Delta}=\underset{h_{2} h_{1}}{\Delta}\right.$ for all $\left.h_{1}, h_{2} \in V\right)$ and if $h \in V$ and $n \in \mathbb{N}$, then $\Delta_{h}^{n}$ - the $n$-th iterate of $\frac{\Delta}{h}$ - satisfies

$$
\Delta_{h}^{n} f(x)=\sum_{k=0}^{n}(-1)^{n-k}\left(\begin{array}{l}
n \\
k
\end{array}\right) f(x+k h) \quad \text { for } f \in B^{V} \text { and } x, h \in V .
$$

The following two theorems were proved by Mazur and Orlicz [12] and [13], and in greater generality by Djoković [5].

Theorem A. If $n \in \mathbb{N}$ and $f: V \rightarrow B$, then the following are equivalent.

(i) $\Delta_{h}^{n} f(x)=0$ for all $x, h \in V$.

(ii) $\underset{h_{n}}{\Delta} \cdots \Delta \underset{h_{1}}{\Delta} f(x)=0$ for all $x, h_{1}, \ldots, h_{n} \in V$.

(iii) $f$ is a GP function of degree at most $n-1$.

The functional equation (i) was (perhaps first) studied by Fréchet [7]. The (Ulam) stability of (i) and (ii) was established by Hyers [9] and by Albert and Baker [1. From Theorem 3 and Lemma 1(b) thereof we have

Theorem B. Suppose that $2 \leq n \in \mathbb{N}, \delta \geq 0, B$ is a Banach space, $f: V \rightarrow B$ and

$$
\left\|\Delta h_{h_{1}} \cdots \underset{h_{n}}{\Delta} f(x)\right\| \leq \delta \quad \text { for all } x, h_{1}, \ldots, h_{n} \in V .
$$

Then there exists a GP function $p: V \rightarrow B$ of degree at most $n-1$ such that $\|f(x)-p(x)\| \leq 2 \delta$ for all $x \in V$.

In [15], Whitney proved that if $V=\mathbb{R}($ or even $(0,+\infty)), f: V \rightarrow \mathbb{R}, \delta>0$,

$$
\left|\Delta_{h}^{n} f(x)\right| \leq \delta \quad \text { for all } x, h \in V
$$

and $f$ is bounded on some interval, then there is a real polynomial function $p$, of degree at most $n-1$, and a constant $k_{n}>0$ such that $|f(x)-p(x)| \leq k_{n} \delta$ for all $x \in V$. 


\section{MAIN RESULTS}

Now let's address the stability of $(*)$. Suppose that $m \in \mathbb{N}, \alpha_{k}, \beta_{k} \in F$ for $0 \leq k \leq m, B$ is a Banach space, $f_{0}, f_{1}, \ldots, f_{m}: V \rightarrow B, \delta \geq 0$ and

$$
\left\|\sum_{k=0}^{m} f_{k}\left(\alpha_{k} x+\beta_{k} y\right)\right\| \leq \delta \quad \text { for all } x, y \in V \text {. }
$$

We assume that $\alpha_{j} \beta_{k}-\alpha_{k} \beta_{j} \neq 0$ whenever $0 \leq j<k \leq m$, i.e. $\left(\alpha_{0}, \beta_{0}\right),\left(\alpha_{1}, \beta_{1}\right)$, $\ldots,\left(\alpha_{m}, \beta_{m}\right)$ are pairwise linearly independent in $F^{2}$. To see why this is a natural assumption, suppose on the contrary (for example) that $\left(\alpha_{m}, \beta_{m}\right)=\gamma\left(\alpha_{m-1}, \beta_{m-1}\right)$ for some $\gamma \in F$. Then $f_{m-1}\left(\alpha_{m-1} x+\beta_{m-1} y\right)+f_{m}\left(\alpha_{m} x+\beta_{m} y\right)=g\left(\alpha_{m-1} x+\beta_{m-1} y\right)$ for all $x, y \in V$ where $g(z)=f_{m-1}(z)+f_{m}(\gamma z)$ for $z \in V$ and thus ( $\dagger$ ) can be written as an inequality involving $m$, rather than $m+1$, functions.

Suppose $m=1$ so that $(\dagger)$ becomes

$$
\left\|f_{0}\left(\alpha_{0} x+\beta_{0} y\right)+f_{1}\left(\alpha_{1} x+\beta_{1} y\right)\right\| \leq \delta, \quad x, y \in V .
$$

Given $u, v \in V$, since $\alpha_{0} \beta_{1}-\alpha_{1} \beta_{0} \neq 0$ there exist unique $x, y \in V$ such that $u=\alpha_{0} x+\beta_{0} y$ and $v=\alpha_{1} x+\beta_{1} y$. Hence

$$
\left\|f_{0}(u)+f_{1}(v)\right\| \leq \delta \quad \text { for all } u, v \in V .
$$

If $c_{0}=-f_{1}(0)$ and $c_{1}=-f_{0}(0)$ we have $\left\|c_{0}+c_{1}\right\| \leq \delta,\left\|f_{0}(u)-c_{0}\right\| \leq \delta$ and $\left\|f_{1}(v)-c_{1}\right\| \leq \delta$ for all $u, v \in V$. Henceforth we therefore assume that $m \geq 2$.

Theorem 1. Suppose that $B$ is a real (or complex) Banach space, $f_{k}: V \rightarrow B$ for $0 \leq k \leq m, \delta>0$ and $(\dagger)$ holds. Then for each $k=0,1, \ldots, m$,

$$
\left\|\Delta{ }_{h_{m}} \cdots \Delta_{h_{1}} f_{k}(x)\right\| \leq 2^{m} \delta \quad \text { for all } x, h_{1}, \ldots, h_{m} \in V
$$

and there exists a GP function $p_{k}: V \rightarrow \mathbb{R}$ and $c_{k} \in B$ such that

$$
\left\|\left(f_{k}(x)-c_{k}\right)-p_{k}(x)\right\| \leq 2^{m+1} \delta \quad \text { for all } x \in V .
$$

Moreover

$$
\sum_{k=0}^{m} p_{k}\left(\alpha_{k} x+\beta_{k} y\right)=0 \quad \text { for all } x, y \in V .
$$

Proof. For $0 \leq j \leq k \leq m$ let $d_{j k}=\alpha_{j} \beta_{k}-\alpha_{k} \beta_{j}$ so that $d_{j k} \neq 0$ if $j<k$ and $d_{k k}=0$.

For $0 \leq k \leq m$ and $x, y, h_{1} \in V$,

$$
\alpha_{k}\left(x+\beta_{m} h_{1}\right)+\beta_{k}\left(y-\alpha_{m} h_{1}\right)=\alpha_{k} x+\beta_{k} y+d_{k m} h_{1}
$$

so that

$$
\left\|\sum_{k=0}^{m} f_{k}\left(\alpha_{k} x+\beta_{k} y+d_{k m} h_{1}\right)\right\|=\| \sum_{k=0}^{m} f_{k}\left(\alpha_{k}\left(x+\beta_{m} h_{1}\right)+\beta_{k}\left(y-\alpha_{m} h_{1}\right) \| \leq \delta .\right.
$$

Using this inequality and $(\dagger)$ we find that

$$
\begin{aligned}
& \left\|\sum_{k=0}^{m}\left\{f_{k}\left(\alpha_{k} x+\beta_{k} y+d_{k m} h_{1}\right)-f_{k}\left(\alpha_{k} x+\beta_{k} y\right)\right\}\right\| \\
& \quad \leq\left\|\sum_{k=0}^{m} f_{k}\left(\alpha_{k} x+\beta_{k} j+d_{k m} h_{1}\right)\right\|+\left\|\sum_{k=0}^{m} f_{k}\left(\alpha_{k} x+\beta_{k} y\right)\right\| \leq 2 \delta
\end{aligned}
$$


and thus, since $d_{m m}=0$,

$$
\left\|\sum_{k=0}^{m-1} \underset{d_{k m} h_{1}}{\Delta} f_{k}\left(\alpha_{k} x+\beta_{k} y\right)\right\| \leq 2 \delta \quad \text { for all } x, y, h_{1} \in V .
$$

Repeating the argument that led from $(\dagger)$ to $(\dagger)_{1}$ we find that

$$
\left\|\sum_{k=0}^{m-2} \underset{d_{k, m-1} h_{2}}{\Delta} \underset{d_{k m} h_{1}}{\Delta} f_{k}\left(\alpha_{k} x+\beta_{k} y\right)\right\| \leq 4 \delta
$$

for all $x, y, h_{1}, h_{2} \in V$.

Applying this reasoning $m-2$ more times, we are inclined to admit that

$$
\left\|\underset{d_{01} h_{m}}{\Delta} \cdots \underset{d_{0 m} h_{1}}{\Delta} f_{0}\left(\alpha_{0} x+\beta_{0} y\right)\right\| \leq 2^{m} \delta \quad \text { for all } x, y, h_{1}, \ldots, h_{m} \in V \text {. }
$$

Since $d_{0 k} \neq 0$ for $1 \leq k \leq m$ and $\left|\alpha_{0}\right|+\left|\beta_{0}\right|>0$, the last inequality simply asserts that

$$
\left\|\underset{h_{m}}{\Delta} \cdots \Delta_{h_{1}} f_{0}(z)\right\| \leq 2^{m} \delta \quad \text { for all } z, h_{1}, \ldots, h_{m} \in V .
$$

By the "symmetry" of our assumptions on $f_{0}, \ldots, f_{m}$, for each $k=0,1, \ldots, m$,

$$
\left\|\Delta{ }_{h_{m}} \cdots \Delta_{h_{1}} f_{k}(x)\right\| \leq 2^{m} \delta \text { for all } x, h_{1}, \ldots, h_{m} \in V,
$$

and hence, according to Theorem $\mathrm{B}$, there exists a GP function $q_{k}: V \rightarrow B$, of degree at most $m-1$, such that

$$
\left\|f_{k}(x)-q_{k}(x)\right\| \leq 2^{m+1} \delta \quad \text { for all } x \in V .
$$

For $0 \leq k \leq m$ and $1 \leq j \leq m-1$ there are symmetric, $j$-additive maps $a_{k j}: V^{j} \rightarrow B$ such that

$$
q_{k}(x)=q_{k}(0)+\sum_{j=1}^{m-1} a_{k j}^{*}(x) \quad \text { for all } x \in V .
$$

By $(\dagger)$ and (1), for all $x, y \in V$,

$$
\begin{aligned}
\left\|\sum_{k=0}^{m} q_{k}\left(\alpha_{k} x+\beta_{k} y\right)\right\| \leq & \left\|\sum_{k=0}^{m}\left\{q_{k}\left(\alpha_{k} x+\beta_{k} y\right)-f_{k}\left(\alpha_{k} x+\beta_{k} y\right)\right\}\right\| \\
& +\left\|\sum_{k=0}^{m} f_{k}\left(\alpha_{k} x+\beta_{k} y\right)\right\| \\
\leq & (m+1) 2^{m+1} \delta+\delta=: \delta_{1} .
\end{aligned}
$$

Now (3) says, in light of (2), that, for all $x, y \in V$,

$$
\left\|\left(\sum_{k=0}^{m} q_{k}(0)\right)+\left(\sum_{j=1}^{m-1} \sum_{k=0}^{m} a_{k j}^{*}\left(\alpha_{k} x+\beta_{k} y\right)\right)\right\| \leq \delta_{1} .
$$

In $(3)^{\prime}$ replace $x$ by $r x$ and $y$ by $r y(r \in \mathbb{Q})$ to conclude that, for all $x, y \in V$ and all $r \in \mathbb{Q}$,

$$
\left\|\left(\sum_{k=0}^{m} q_{k}(0)\right)+\sum_{j=1}^{m-1} r^{j}\left(\sum_{k=0}^{m} a_{k j}^{*}\left(\alpha_{k} x+\beta_{k} y\right)\right)\right\| \leq \delta_{1} .
$$

"By continuity", (4) holds for all real $r$, and all $x, y \in V$. 
Now suppose that $\Lambda: B \rightarrow \mathbb{R}$ is a continuous linear functional. Then by (4),

$$
\left|\Lambda\left(\sum_{k=0}^{m} q_{k}(0)\right)+\sum_{j=1}^{m-1} r^{j} \Lambda\left\{\sum_{k=0}^{m} a_{k j}^{*}\left(\alpha_{k} x+\beta_{k} y\right)\right\}\right| \leq\|\Lambda\| \delta_{1}
$$

for all $x, y \in V$ and all $r \in \mathbb{R}$.

Since a real polynomial function is bounded if and only if it is constant, from the last inequality we surmise that, for $1 \leq j \leq m-1$,

$$
\Lambda\left\{\sum_{k=0}^{m} a_{k j}^{*}\left(\alpha_{k} x+\beta_{k} y\right)\right\}=0 \quad \text { for all } x, y \in V .
$$

Since this is so for every continuous linear functional $\Lambda: B \rightarrow \mathbb{R}$, by the HahnBanach theorem,

$$
\sum_{k=0}^{m} a_{k j}^{*}\left(\alpha_{k} x+\beta_{k} y\right)=0 \quad \text { for } x, y \in V \text { and } 1 \leq j \leq m-1 .
$$

Let $p_{k}(x)=q_{k}(x)-q_{k}(0)=\sum_{j=1}^{m-1} a_{k j}^{*}(x), x \in V$, for $0 \leq k \leq m$. Then each $p_{k}$ is a GP function of degree at most $m-1$ and from (5) we find that

$$
\sum_{k=0}^{m} p_{k}\left(\alpha_{k} x+\beta_{k} y\right)=0 \quad \text { for all } x, y \in V .
$$

Finally, letting $c_{k}=q_{k}(0)$ for $0 \leq k \leq m$, we find from (1) that if $0 \leq k \leq m$, then

$$
\left\|\left(f_{k}(x)-c_{k}\right)-p_{k}(x)\right\| \leq\left\|f_{k}(x)-q_{k}(x)\right\| \leq 2^{m+1} \delta \quad \text { for all } x \in V .
$$

Note that the possibility that $\delta=0$ has not been excluded. Thus the solutions of $(*)$ are GP functions of degree at most $m-1$.

\section{REGULARITY PROPERTIES OF $(*)$ AND $(\dagger)$}

The following result is a special case of Theorem 6.1 (iii) of Kemperman 10].

Theorem C. Suppose that $(\dagger)$ holds where $F=\mathbb{R}, V=\mathbb{R}^{n}$ and $B=\mathbb{C}$. If some $f_{k}$ is bounded on a subset of $\mathbb{R}^{n}$ having positive inner Lebesgue measure, then $p_{k}$ is a genuine polynomial function.

\section{A MEAN VALUE THEOREM}

The next theorem comes from Walsh [14]; also see [3].

Theorem D. If $2 \leq m \in \mathbb{N}, f: \mathbb{R}^{2} \rightarrow \mathbb{R}$ and $f$ is continuous, then the following are equivalent.

(i) For every regular m-gon in $\mathbb{R}^{2}$, the value of $f$ at its center is the arithmetic mean of its value at the vertices.

(ii) The function $f$ is a harmonic polynomial of degree at most $m-1$. 
If we let $\beta_{k}=\exp (2 \pi k i / m)$ for $1 \leq k \leq m$ and identify $\mathbb{R}^{2}$ with $\mathbb{C}$, then (i) asserts that

$$
\sum_{k=1}^{m} f\left(z+\beta_{k} w\right)=m f(z) \quad \text { for all } z, w \in \mathbb{C} .
$$

This leads us to

Theorem 2. Suppose that $2 \leq m \in \mathbb{N}, f: \mathbb{C} \rightarrow \mathbb{C}, \delta \geq 0, \beta_{k}=\exp (2 \pi k i / m)$ for $1 \leq k \leq m$ and

$$
\left|m f(z)-\sum_{k=1}^{m} f\left(z+\beta_{k} w\right)\right| \leq \delta \quad \text { for all } z, w \in \mathbb{C} .
$$

Then there exists a $c \in \mathbb{C}$ and a $G P$ function $p: \mathbb{C} \rightarrow \mathbb{C}$, of degree at most $m-1$, such that

$$
\begin{array}{cc}
|f(z)-c-p(z)| \leq 2^{m+1} \delta & \text { for all } z \in \mathbb{C} \text { and } \\
m p(z)=\sum_{k=1}^{m} p\left(z+\beta_{k} w\right) & \text { for all } z, w \in \mathbb{C} .
\end{array}
$$

Moreover, if $f$ is bounded on some subset of $\mathbb{C}\left(=\mathbb{R}^{2}\right)$ having positive inner Lebesgue measure, then $p$ is a harmonic polynomial of degree at most $m-1$.

Proof. By putting $f_{0}=m f$ and $f_{k}=-f$ for $1 \leq k \leq m$ and letting $\beta_{0}=0$ and $\alpha_{k}=1$ for $0 \leq k \leq m$, we deduce from Theorem 1 that there exist $c_{0}, c \in \mathbb{C}$ and GP functions $p_{0}, p: \mathbb{C} \rightarrow \mathbb{C}$, each of degree at most $m-1$, such that

$$
\begin{aligned}
& \left|f_{0}(z)-c_{0}-p_{0}(z)\right| \leq 2^{m+1} \delta \text { and } \\
& |f(z)-c-p(z)| \leq 2^{m+1} \delta \text { for all } z \in \mathbb{C},
\end{aligned}
$$

and moreover

$$
m p_{0}(z)=\sum_{k=1}^{m} p\left(z+\beta_{k} w\right) \quad \text { for all } z, w \in \mathbb{C} .
$$

But when $w=0$ this last equation tells us that $p_{0}=p$.

By Theorem 1, $\left|\underset{h_{m}}{\Delta} \cdots \underset{h_{1}}{\Delta} f(z)\right| \leq 2^{m} \delta$ for all $z, h_{1}, \ldots, h_{m} \in \mathbb{C}$. Hence, for all $z, h \in \mathbb{C}$,

$$
\left|\sum_{k=0}^{m}(-1)^{m-k}\left(\begin{array}{c}
m \\
k
\end{array}\right) f(z+k h)\right|=\left|\Delta_{h}^{n} f(z)\right| \leq 2^{m} \delta
$$

and therefore, by Theorem $\mathrm{C}$, if $f$ is bounded on some subset of $\mathbb{C}=\mathbb{R}^{2}$ of positive inner Lebesgue measure, then $p$ is an ordinary polynomial function on $\mathbb{R}^{2}$. In this case, Theorem D implies that $p$ is harmonic.

\section{A Distributional AnAlogue of $(*)$}

Suppose that $I$ and $J$ are open intervals in $\mathbb{R}, \alpha_{0}, \beta_{0}, \ldots, \alpha_{m}, \beta_{m} \in \mathbb{R}$ with $\alpha_{j} \beta_{k}-\alpha_{k} \beta_{j} \neq 0$ for $0 \leq j<k \leq m, I_{k}=\left\{\alpha_{k} x+\beta_{k} y: x \in I, y \in J\right\}$ for $0 \leq k \leq m, f_{k}: I_{k} \rightarrow \mathbb{C}$ for $0 \leq k \leq m, g_{j}: I \rightarrow \mathbb{C}$ and $h_{j}: J \rightarrow \mathbb{C}$ for $1 \leq j \leq n$ and

$$
\sum_{k=0}^{m} f_{k}\left(\alpha_{k} x+\beta_{k} y\right)=\sum_{j=1}^{n} g_{j}(x) h_{j}(y) \quad \text { for }(x, y) \in I \times J .
$$


In 22 it was shown that if the $f_{k}$ 's are locally Lebesgue integrable, then they are restrictions to $I_{k}$ of exponential polynomials. It follows that the locally integrable solutions of $(*)$ are polynomial functions.

Let $T_{k}(x, y)=\alpha_{k} x+\beta_{k} y$ for $(x, y) \in I \times J$. Then $(*)$ holds (for $x \in I$ and $y \in J$ ) if and only if

$$
\sum_{k=0}^{m} f_{k} \circ T_{k}=0
$$

Now each $T_{k}$ is a submersion of $I \times J$ onto $I_{k}$ - see Chapter VI of Hormander [8] and if $u \in \mathcal{D}^{\prime}\left(I_{k}\right)$, there is a member $T_{k}^{*} u$ of $\mathcal{D}^{*}(I \times J)$, called the pullback of $u$ by $T_{k}$, which serves as a suitable generalization of "composition". In [2] we used the more suggestive notation $u \circ T$ in place of the more popular $T^{*} u$. Thus a natural question to ask, concerning $(*)$, in the realm of Schwartz distribution theory is, for which $u_{k} \in \mathcal{D}^{\prime}\left(I_{k}\right), 0 \leq k \leq m$ is it true that

$$
\sum_{k=0}^{m} u_{k} \circ T_{k}=0
$$

It follows from Theorem 3 of [2, wherein a distributional analogue of (6) was considered, that if $(* *)$ holds, then each $u_{k}$ is a regular distribution corresponding to a polynomial function $p_{k}: \mathbb{R} \rightarrow \mathbb{C}$ of degree at most $m-1$.

\section{RELATED CONVEXITY RESULTS}

If $I$ is an interval in $\mathbb{R}$ and $f: I \rightarrow \mathbb{R}$, then $f$ is said to be midpoint convex or Jensen convex provided $f\left(\frac{x+y}{2}\right) \leq \frac{f(x)+f(y)}{2}$ for all $x, y \in I$; equivalently,

$$
f(x)-2 f(x+h)+f(x+2 h)=\Delta_{h}^{2} f(x) \geq 0
$$

whenever $h>0, x \in I$ and $x+2 h \in I$.

A function $f: I \rightarrow \mathbb{R}$ is said to be (Jensen) convex of order $n-1$ provided $\Delta_{h}^{n} f(x) \geq 0$ whenever $h>0, x \in I$ and $x+n h \in I$. Ciesielski proved in [4] that if such a function is bounded on a subset of $I$ having positive Lebesgue inner measure, then it is continuous on $I$. This result was generalized in Kemperman [11.

A survey of stability results concerning functional equations can be found in [6]. Numerous other papers on the subject have been published in the journal Aequationes Mathematicae in the last two decades.

\section{REFERENCES}

1. Michael Albert and John A. Baker, Functions with bounded nth differences, Ann. Polon. Math. 43 (1983), 93-103. MR0727892 (85m:39001)

2. John A. Baker, On a functional equation of Aczél and Chung, Aeq. Math. 46 (1993), 99-111. MR1220725 (95e:39004)

3. John A. Baker, Mean value theorems via spectral synthesis, J. Math. Anal. Appl. 193 (1995), 306-317. MR1338515 (96k:39028)

4. Z. Ciesielski, Some properties of convex functions of higher orders, Ann. Polon. Math. 7 (1959), 1-7. MR0109202(22:89)

5. D.Ž. Djoković, A representation theorem for $\left(X_{1}-1\right)\left(X_{2}-1\right) \cdots\left(X_{n}-1\right)$ and its applications, Ann. Polon. Math. 22 (1969), 189-198. MR0265798(42:707)

6. G.L. Forti, Hyers-Ulam stability of functional equations in several variables, Aequationes Math. 50 (1995), 143-190. MR.1336866 (96i:39033)

7. M. Fréchet, Pri la funkcia equacio $f(x+y)=f(x)+f(y)$, Enseignement Math. 15 (1913), 390-393. 
8. Lars Hormander, The analysis of linear partial differential operators. I, Second Edition, Springer-Verlag, Berlin, 1990. MR.1065993 (91m:35001a)

9. D.H. Hyers, Transformations with bounded n-th differences, Pacific J. Math. 11 (1961), 591602. MR0132401 (24:A2246)

10. J.H.B. Kemperman, A general functional equation, Trans. Amer. Math. Soc. 86 (1957), $28-56$. MR0094610 (20:1123)

11. J.H.B. Kemperman, On the regularity of generalized convex functions, Trans. Amer. Math. Soc. 135 (1969), 69-93. MR 0265531(42:440) MR0232900|(38:1223)

12. S. Mazur and W. Orlicz, Grundlegende Eigenschaften der Polynomischen Operationen, Erst Mitteilung, Studia Math. 5 (1934), 50-68.

13. S. Mazur and W. Orlicz, Grundlegende Eigenschaften der Polynomischen Operationen, Zweite Mitteilung, ibidem 5 (1934), 179-189.

14. J.L. Walsh, A mean value theorem for polynomials and harmonic polynomials, Bull. Amer. Math. Soc. (1936), 923-930.

15. H. Whitney, On functions with bounded n-th differences, J. Math. Pures Appl. (9) 36 (1957), 67-95. MR0084611 (18:889f)

Department of Pure Mathematics, University of Waterloo, Waterloo, Ontario, CANADA N2L 3G1

E-mail address: jabaker@math.uwaterloo.ca 